\title{
Camera Identification from Cropped and Scaled Images
}

\author{
Miroslav Goljan* and Jessica Fridrich \\ Department of Electrical and Computer Engineering \\ SUNY Binghamton, Binghamton, NY 13902-6000
}

\begin{abstract}
In this paper, we extend our camera identification technology based on sensor noise to a more general setting when the image under investigation has been simultaneously cropped and scaled. The sensor fingerprint detection is formulated using hypothesis testing as a two-channel problem and a detector is derived using the generalized likelihood ratio test. A brute force search is proposed to find the scaling factor which is then refined in a detailed search. The cropping parameters are determined from the maximum of the normalized cross-correlation between two signals. The accuracy and limitations of the proposed technique are tested on images that underwent a wide range of cropping and scaling, including images that were acquired by digital zoom. Additionally, we demonstrate that sensor noise can be used as a template to reverse-engineer in-camera geometrical processing as well as recover from later geometrical transformations, thus offering a possible application for re-synchronizing in digital watermark detection.
\end{abstract}

Keywords: Camera identification, Photo-Response Non-Uniformity, digital forensic.

\section{INTRODUCTION}

The problem of establishing the origin of digital media obtained through an imaging sensor has received significant attention in the last few years. ${ }^{1-12}$ A successful solution to this problem will find immediate applications in situations when digital content is presented as silent witness in the court. For example, in a child pornography case, establishing solid evidence that a given image was obtained using a suspect's camera is obviously very important. The identification technology could also be used to link a camcorder confiscated inside a movie theater to other, previously pirated content.

In these applications, it is necessary to link an image or a video-clip to a specific piece of hardware. Sensor photoresponse non-uniformity (PRNU) has been previously proposed" as an equivalent of "biometrics for sensors". There are several important advantages of using this fingerprint for forensic purposes:

a) Stability. The fingerprint is stable in time and under a wide range of physical conditions.

b) Generality. The fingerprint is present in every picture (with the exception of completely dark images) independently of the camera optics, settings, or the scene content.

c) Universality. Virtually all sensors exhibit PRNU (both CCD and CMOS sensors).

d) Dimensionality. The fingerprint has large information content because it is a signal that contains a stochastic component due to material properties of silicone and the manufacturing process itself. Thus, it is unlikely that two sensors will possess similar fingerprints.

e) Robustness. The fingerprint survives a wide range of common image processing operations, including lossy compression, filtering, and gamma adjustment.

One of the disadvantages of PRNU when used as camera/sensor fingerprint is that its detection is very sensitive to proper synchronization. If the image under investigation has been slightly cropped or scaled, the direct detection will

*mgoljan@binghamton.edu; phone +001-607-777-5793; fax +001-607-777-4464. 
not succeed. The problem with geometrical transformations is well known to the watermarking community and is usually solved by a special watermark/template design by either making the watermark self-referencing, ${ }^{13}$ symmetrical, ${ }^{14}$ or by switching to a domain ${ }^{15}$ invariant to rotation, scaling, and translation. In forensic applications, however, the fingerprint (the "watermark") is not our choice and is imposed on us rather than designed. Thus, we cannot expect it to have any properties that would make the detection easier. Moreover, the energy of a typical robust watermark is likely to be by several orders of magnitude larger than the energy of PRNU. Consequently, it is substantially more difficult to detect the fingerprint after geometrical processing than it is for a human-designed digital watermark.

Geometrical transformations, such as scaling or rotation, cause desynchronization and introduce additional distortion due to resampling. There are fundamentally at least three different approaches to attack this problem. The first possibility is to use methods for blind estimation of resampling parameters ${ }^{16}$ invert the geometrical transformation, and detect the sensor fingerprint. However, as reported in [16] these methods do not survive well JPEG compression (JPEG quality factor of 97 is already significantly reducing the performance). The second possibility is to use the Fourier-Mellin transform ${ }^{15}$ that solves the problem of recovering the scaling and rotation parameters using a simple cross-correlation in the log-polar domain. The non-linear resampling needed to transform the image, however, introduces additional distortion, which in our case is a serious factor because the SNR between the sensor fingerprint and the image is $-50 \mathrm{~dB}$ or less. In this paper we focus on the third option - using the Generalized Likelihood Ratio Test directly in the spatial domain and finding the maximum of the test statistics using brute force.

We constrain ourselves to geometrical transformations that only include scaling and cropping, leaving out the rotation by an arbitrary angle. This choice is motivated by an anecdotal survey that the authors performed on their students and colleagues. The result of this survey was that most people do not process their images and those who do are almost always satisfied with cropping the image or resizing it to a smaller scale before sending via e-mail or archiving. Most typical users rarely rotate their images by other amount than by 90 degrees, which can be easily incorporated into the proposed approach without increasing its complexity in any significant manner. Skipping the rotation simplifies the brute force search for the correct transform parameters and enables relatively efficient camera ID from images that were simultaneously cropped and scaled. In the next section, we describe our approach and in Section 3, we report the results of experiments. The last section contains a summary and an outline for future research.

Everywhere in this paper, boldface font will denote vectors or matrices of length specified in the text, e.g., $\mathbf{X}[i]$ and and $\mathbf{Y}[i, j]$ denote the $i$-th component of vector $\mathbf{X}$ and $i, j$-th element of matrix $\mathbf{Y}$, respectively. Unless mentioned otherwise, all operations among vectors or matrices, such as product, ratio, raising to a power, etc., are element-wise. The norm of $\mathbf{X}$ is denoted as $\|\mathbf{X}\|=\sqrt{\mathbf{X} \odot \mathbf{X}}$ with $\mathbf{X} \odot \mathbf{Y}=\sum_{i=1}^{n} \mathbf{X}[i] \mathbf{Y}[i]$ being the dot product of two vectors. Denoting the mean values with a bar, the normalized cross-correlation (NCC) between $\mathbf{X}$ and $\mathbf{Y}$ is a matrix

$$
\mathbf{N C C}[i, j]=\frac{\sum_{k=1}^{m} \sum_{l=1}^{n}(\mathbf{X}[k, l]-\overline{\mathbf{X}})(\mathbf{Y}[k+i, l+j]-\overline{\mathbf{Y}})}{\|\mathbf{X}-\overline{\mathbf{X}}\| \mathbf{Y}-\overline{\mathbf{Y}} \|} .
$$

If $\mathbf{X}$ and $\mathbf{Y}$ have different dimensions, they are padded with zeros on the right and at the bottom.

\section{CAMERA IDENTIFICATION AS TWO-CHANNEL PROBLEM}

In this section, we describe the general methodology for camera identification from images that were simultaneously cropped and resized. We start by formulating the problem using hypothesis testing and derive optimal and suboptimal detectors from a camera output model.

We start by describing the cropping and scaling process. Let us assume that the original image is in grayscale represented with an $m \times n$ matrix $\mathbf{I}[i, j] \in\{0,1, \ldots, 255\}, i=1, \ldots, m, j=1, \ldots, n$. Let us denote as $\mathbf{u}=\left(u_{r}, u_{l}, u_{t}, u_{b}\right)$ the vector describing the cropping process, where the non-negative integers $u_{r}, u_{l}, u_{t}$, and $u_{b}$ stand for the number of pixels cropped out from the image from the right, left, top, and bottom, respectively. Note that when the cropped image and the original image are aligned by their upper left corner, the cropping process introduces a spatial translation $T_{\mathbf{s}}$ by the vector $\mathbf{s}=\left(u_{l}, u_{t}\right)$ with respect to the original image in the sense that for the cropped image $\mathbf{Y}$, 
$\mathbf{Y}[i, j]=\mathbf{I}\left[i+u_{l}, j+u_{t}\right]$ for all $i=1, \ldots, m-u_{r}-u_{l}, j=1, \ldots, n-u_{t}-u_{b}$. By subsequently downscaling the cropped image by the factor of $r, 0<r \leq 1$, we obtain the image $\mathbf{Z}[i, j], i=1, \ldots, M, j=1, \ldots, N$, where $M=r\left(m-u_{r}-u_{l}\right)$ and $N=r(n-$ $\left.u_{t}-u_{b}\right)$. Here, we assume that $M$ and $N$ are integers. The resulting transformation, which is a composition consisting of a spatial shift and subsequent scaling $T_{r} \circ T_{\mathrm{s}}$, will thus have one vector parameter $\mathbf{s}$ and one scalar parameter $r$

$$
\mathbf{Z}=\left(T_{r} \circ T_{\mathrm{s}}\right)(\mathbf{I})=T_{r}\left(T_{\mathrm{s}}(\mathbf{I})\right) .
$$

The precise mechanism of $T_{r}$ depends on the downsampling algorithm.

The problem we are facing is to determine whether image $\mathbf{Z}$ was taken with a given camera, whose fingerprint is known. As in [17], it is advantageous to work with the noise residual $\mathbf{W}$ of images obtained using a denoising filter ${ }^{\dagger}$ $F, \mathbf{W}(\mathbf{I})=\mathbf{I}-F(\mathbf{I})$. We use a simplified linearized model for the noise residual as described in [17],

$$
\mathbf{W}(\mathbf{I})=\mathbf{I}-F(\mathbf{I})=\mathbf{I K}+\mathbf{\Xi},
$$

where IK is the PRNU term with $\mathbf{K}$ being the multiplicative PRNU factor (camera fingerprint) and $\boldsymbol{\Xi}$ is a noise term. The noise residual of the cropped/scaled image is thus the geometrically transformed PRNU term + noise term: $\mathbf{W}(\mathbf{Z})=T_{r} \circ T_{\mathbf{s}}(\mathbf{I K})+\boldsymbol{\Xi}$. The task of deciding whether or not a camera with fingerprint $\mathbf{K}$ took the image $\mathbf{I}$ can thus be formulated as a two-channel hypothesis testing problem that we state in a slightly more general form

$$
\begin{aligned}
& \mathrm{H}_{0}: \mathbf{K} \neq \mathbf{K}^{\prime} \\
& \mathrm{H}_{1}: \mathbf{K}=\mathbf{K}^{\prime}
\end{aligned}
$$

where

$$
\begin{aligned}
& \hat{\mathbf{K}}=\mathbf{K}+\mathbf{\Xi}_{1} \\
& \mathbf{W}=T_{r} \circ T_{\mathbf{s}}\left(\mathbf{I K}^{\prime}+\boldsymbol{\Xi}_{2}\right)
\end{aligned}
$$

are two observed signals; $\hat{\mathbf{K}}$ is the camera PRNU estimated using the techniques described in [17], $\mathbf{K}^{\prime}$ is the PRNU from the camera that took the image, and $\boldsymbol{\Xi}_{1}, \boldsymbol{\Xi}_{2}$ are noise terms. The signal $\mathbf{W}$ is the rescaled and shifted noise residual of the image under investigation. We note that $r$ and $\mathbf{s}$ are unknown parameters that also need to be estimated.

The noise is modeled as a sequence of zero-mean i.i.d. Gaussian variables with known variances $\sigma_{1}^{2}, \sigma_{2}^{2}$. The optimal detector for this two-channel model has been derived by Holt ${ }^{19}$. The test statistics, obtained using the generalized likelihood ratio test is a sum of three terms: two energy-like quantities and a cross-correlation term:

$$
t=\max _{r, \mathbf{s}}\left\{E_{1}(r, \mathbf{s})+E_{2}(r, \mathbf{s})+C(r, \mathbf{s})\right\}
$$

with

$$
\begin{aligned}
& E_{1}(r, \mathbf{s})=\sum_{i, j} \frac{\left(\hat{\mathbf{K}}\left[i+s_{1}, j+s_{2}\right]\right)^{2}}{\sigma_{1}^{2}+\sigma_{1}^{4} \sigma_{2}^{-2}\left(T_{1 / r}(\mathbf{Z})\left[i+s_{1}, j+s_{2}\right]\right)^{2}}, \\
& E_{2}(r, \mathbf{s})=\sum_{i, j} \frac{\left(T_{1 / r}(\mathbf{Z})\left[i+s_{1}, j+s_{2}\right]\right)^{2}\left(T_{1 / r}(\mathbf{W})\left[i+s_{1}, j+s_{2}\right]\right)^{2}}{\sigma_{2}^{2}\left(T_{1 / r}(\mathbf{Z})\left[i+s_{1}, j+s_{2}\right]\right)^{2}+\sigma_{2}^{4} \sigma_{1}^{-2}}, \\
& C(r, \mathbf{s})=\sum_{i, j} \frac{\hat{\mathbf{K}}[i, j]\left(T_{1 / r}(\mathbf{Z})\left[i+s_{1}, j+s_{2}\right]\right)\left(T_{1 / r}(\mathbf{W})\left[i+s_{1}, j+s_{2}\right]\right)^{2}}{\sigma_{2}^{2}+\sigma_{1}^{2}\left(T_{1 / r}(\mathbf{Z})\left[i+s_{1}, j+s_{2}\right]\right)^{2}} .
\end{aligned}
$$

Unfortunately, the complexity of evaluating these three expressions is prohibitive. It is proportional to the square of the number of pixels because they cannot be evaluated using the fast Fourier transform as a simple cross-correlation

\footnotetext{
${ }^{\dagger}$ We use a wavelet based denoising filter that removes from images Gaussian noise with variance $\sigma_{F}^{2}$ (we used $\left.\sigma_{F}^{2}=4\right)$. See [18] for more details about this filter.
} 
can. Due to the unfeasibly large computational requirements of this optimal detector, we decided to use a very fast suboptimal detector instead.

The energy terms $E_{1}$ and $E_{2}$ vary only little and slowly with $\mathbf{s}$. The maximum in (5) is due to the contribution of the cross-correlation term that exhibits a sharp peak for the proper values of the geometrical transformation when the image is from the camera. Thus, we selected as an alternative test statistics the following NCC between $\mathbf{X}$ and $\mathbf{Y}$, which is suboptimal but easily calculable using FFT

$$
\mathbf{N C C}\left[s_{1}, s_{2} ; r\right]=\frac{\sum_{k=1}^{m} \sum_{l=1}^{n}(\mathbf{X}[k, l]-\overline{\mathbf{X}})\left(\mathbf{Y}\left[k+s_{1}, l+s_{2}\right]-\overline{\mathbf{Y}}\right)}{\|\mathbf{X}-\overline{\mathbf{X}}\|\|\mathbf{Y}-\overline{\mathbf{Y}}\|},
$$

where

$$
\mathbf{X}=\frac{\hat{\mathbf{K}}}{\sqrt{\sigma_{2}^{2}+\sigma_{1}^{2}\left(T_{1 / r}(\mathbf{Z})\right)^{2}}}, \mathbf{Y}=\frac{T_{1 / r}(\mathbf{Z}) T_{1 / r}(\mathbf{W})}{\sqrt{\sigma_{2}^{2}+\sigma_{1}^{2}\left(T_{1 / r}(\mathbf{Z})\right)^{2}}},
$$

Notice that NCC is an $m \times n$ matrix parametrized by $r$. The noise term for the estimated camera PRNU is much weaker compared to the noise term for the noise residual of the image under investigation. Thus, $\sigma_{1}^{2}=\operatorname{var}\left(\boldsymbol{\Xi}_{1}\right) \ll \operatorname{var}\left(\boldsymbol{\Xi}_{2}\right)=\sigma_{2}^{2}$ and we can further simplify the detector (6) by using $\mathbf{X}=\hat{\mathbf{K}}$ and $\mathbf{Y}=T_{1 / r}(\mathbf{Z}) T_{1 / r}(\mathbf{W})$.

\subsection{Search for the parameters of the geometrical transformation}

The dimensions of the image under investigation, $M \times N$, determine the search range for both the scaling and cropping parameters. We search for the scaling parameter at discrete values $r_{i} \leq 1, i=0,1, \ldots, R$, from $r_{0}=1$ (no scaling, just cropping) down to $r_{\min }=\max \{M / m, N / n\}<1$. For a fixed scaling parameter $r_{i}$, the cross-correlation (6) does not have to be computed for all shifts $\mathbf{s}$ but only for those that move the upsampled image $T_{1 / r_{i}}(\mathbf{Z})$ within the dimensions of the PRNU because only such shifts can be generated by cropping. Given that the dimensions of the upsampled image $T_{1 / r_{i}}(\mathbf{Z})$ are $M / r_{i} \times N / r_{i}$, we have the following range for the spatial shift $\mathbf{s}=\left(s_{1}, s_{2}\right)$

$$
0 \leq s_{1} \leq m-M / r_{i} \text { and } 0 \leq s_{2} \leq n-N / r_{i} \text {. }
$$

To find the maximum value in the GLRT, we evaluate the two-dimensional NCC for each $r_{i}$ as a function of the spatial shift $\mathbf{s}$, and calculate some measure of its peak. There exist several different measures of peak sharpness. ${ }^{20}$ In this paper, we chose the Peak to Correlation Energy measure defined as

$$
\operatorname{PCE}\left(r_{i}\right)=\frac{\mathbf{N C C}\left[\mathbf{s}_{\text {peak }} ; r_{i}\right]^{2}}{\frac{1}{m n-|\mathcal{N}|} \sum_{\mathbf{s}, \mathbf{s} \notin \mathcal{N}} \mathbf{N C C}\left[\mathbf{s} ; r_{i}\right]^{2}},
$$

where $\mathcal{N}$ is a small region surrounding the peak value. As the output of the search, we plot PCE $\left(r_{i}\right)$ as a function of $r_{i}$. If the maximum of $\operatorname{PCE}\left(r_{i}\right)$ is above a threshold $\tau$ we decide $\mathrm{H}_{1}$. The value of the scaling parameter at which the PCE attains this maximum determines the scaling ratio $r_{\text {peak }}$. The location of the peak $\mathbf{s}_{\text {peak }}$ in the $2 \mathrm{D}$ normalized crosscorrelation determines the cropping parameters. Thus, as a by-product of this algorithm, we can determine the processing history of the image under investigation (see Fig. 1b). The PRNU plays the role of a synchronization template similar to templates used in digital watermarking and, in fact, can be used for this purpose when recovering watermark from a geometrically transformed image.

The threshold $\tau$ depends on a user defined upper bound on the false alarm rate and the size of the search space, which is in turn determined by the dimensions of the image under investigation (see Section 2.2).

We now describe in more detail the sampling values $r_{i}$ used in the search for the scaling ratio. The true peak has a certain finite thickness as a result of kernel filtering when acquiring the image due to demosaicking (color interpolation) and possibly the nature of the resampling algorithm itself. We take advantage of this and proceed in two 
phases that increase the speed of the search. In the first (coarse) phase of this brute force search, the sampling values $r_{i}<1$ are

$$
r_{i}=\frac{1}{1+0.005 i}, i=0,1,2, \ldots
$$

In other words, the decrement in the scaling ratio is $0.5 \%$ when $r \sim 1$ and becomes smaller as $r_{i}$ approaches $r_{\min }$ from above. The coarse search first inspects the neighborhood of the scaling ratio corresponding to the case when only scaling and no cropping was applied. Then, we search for $r_{0}, r_{1}, r_{2}, \ldots$, starting with the "cropping only case" or $r_{0}=1$. If we encounter PCE that is significantly higher than what can be expected by pure chance, we stop as soon as $\operatorname{PCE}\left(r_{j+1}\right)<\operatorname{PCE}\left(r_{j}\right)$. This arrangement allows us to reduce the search time when either only cropping or scaling is applied. In the second (refined) phase we improve the accuracy of the scaling ratio estimate by a finer search in the interval $\left(r_{j+1}, r_{j-1}\right)$. A detailed pseudo-code for this search algorithm is given below. As an illustrative example, in Fig. 1 (top) we show PCE $\left(r_{i}\right)$ from a full search for all $r_{i}$ given by (9) as dots. Crosses depict the second refined search phase.
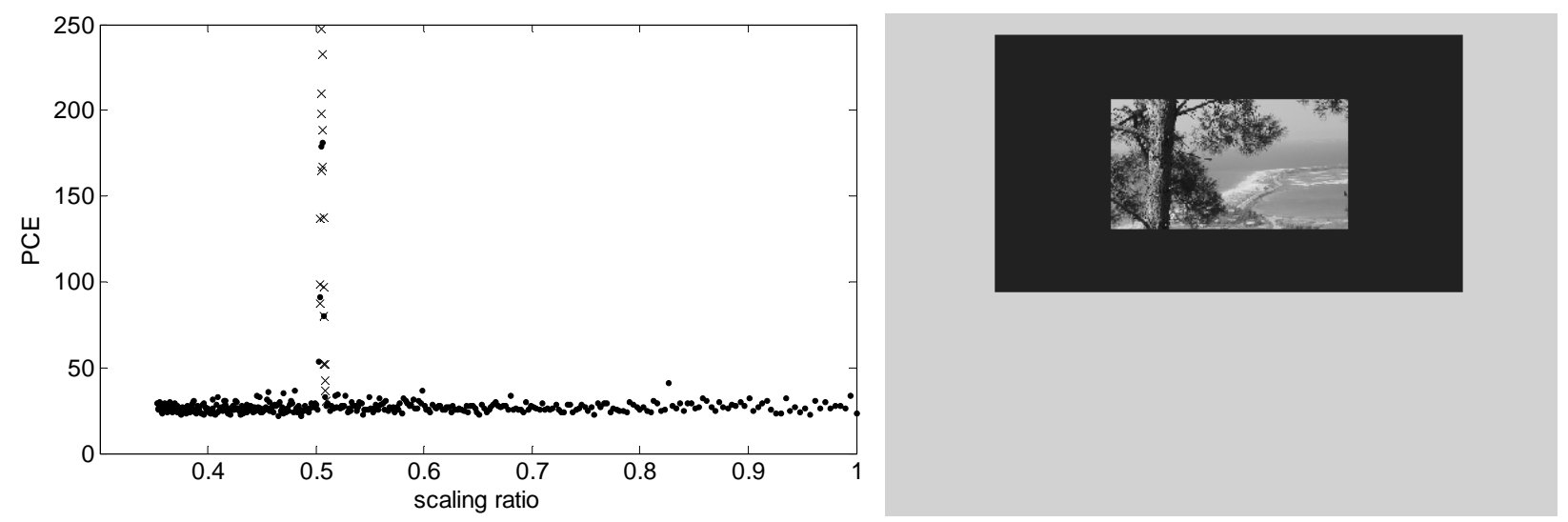

Fig. 1. Left: Detected peak in PCE $\left(r_{i}\right)$. Dots correspond to the first phase, crosses to the second phase of the search. Right: Visual representation of the detected cropping and scaling parameters $r_{\text {peak }}$ and $\mathbf{s}_{\text {peak }}$. The gray frame reflects the original image size, while the black frame shows the image size after cropping before resizing.

We now summarize our implementation of the detection and search algorithm using the following pseudo-code. Here we use the "grayscale" PRNU estimate $\hat{\mathbf{K}}=0.2989 \times \hat{\mathbf{K}}_{\mathrm{R}}+0.5870 \times \hat{\mathbf{K}}_{\mathrm{G}}+0.1140 \times \hat{\mathbf{K}}_{\mathrm{B}}$ obtained as a linear combination of the PRNU signals estimated from each color channel. To reduce the amount of operations, we replaced $T_{1 / r}(\mathbf{Z}) T_{1 / r}(\mathbf{W})$ with $T_{1 / r}(\mathbf{Z W})$. These two terms are equal when $T$ is the nearest-neighbor interpolation method, which is the one we used. Bilinear interpolation, surprisingly, did not perform as efficiently as the nearest-neighbor method in most experiments.

1. Read True color image $\mathbf{Z}$, with $M$ rows and $N$ columns of pixels.

$\mathrm{PCE}_{\text {peak }}=0$; $\hat{\mathbf{K}}$ is the estimated PRNU with $m$ rows and $n$ columns.

2. Set $\quad r_{\min }=\max \{M / m, N / n\}$

$$
\mathbf{r}=\left(r_{0}, r_{1}, r_{2}, \ldots, r_{\min }\right), r_{i}=\frac{1}{1+0.005 i}, i=0,1,2, \ldots, R-1 ;
$$

$\tau \quad$ ddetection threshold for PCE for a given $P_{\mathrm{FA}}$ (see Section 2.2)\}

3. Extract noise from $\mathbf{Z}$ in each color channel and combine the matrices using a linear transform (weighted as luminance) to obtain $\mathbf{W}=0.2989 \times \mathbf{W}_{\mathrm{R}}+0.5870 \times \mathbf{W}_{\mathrm{G}}+0.1140 \times \mathbf{W}_{\mathrm{B}}$.

4. Convert $\mathbf{Z}$ to grayscale.

5. For $i=(R-3, R-2, R-1,0,1, \ldots, R-4)$

begin \{phase 1 \} 
6. Up-sample noise $\mathbf{W}$ by factor $1 / r_{i}$ to obtain $T_{1 / r_{i}}(\mathbf{W})$ (nearest neighbor algorithm used).

7. Calculate the NCC matrix (6) with $\mathbf{X}=\hat{\mathbf{K}}$ and $\mathbf{Y}=T_{1 / r_{i}}(\mathbf{Z}) T_{1 / r_{i}}(\mathbf{W})$.

8. Obtain PCE $\left(r_{i}\right)$ according to (8).

9. If $\operatorname{PCE}\left(r_{i}\right)>\mathrm{PCE}_{\text {peak }}$, then $\operatorname{PCE} E_{\text {peak }}=\operatorname{PCE}\left(r_{i}\right) ; j=i$;

end

elseif $\mathrm{PCE}_{\text {peak }}>\tau$ then go to Step 10 .

end $\quad$ pphase 1 \}

10. Set $\quad r_{\text {step }}=1 / \max \{m, n\} ; \mathbf{r}^{\prime}=\left(r_{j-1}-r_{\text {step }}, r_{j-1}-2 r_{\text {step }}, \ldots, r_{j+1}+r_{\text {step }}\right)=\left(r_{1}^{\prime}, r_{2}^{\prime}, \ldots, r_{R^{\prime}}^{\prime}\right)$;

11. For $i=\left(1, \ldots, R^{\prime}\right)$

begin \{phase 2 \}

12. Up-sample noise $\mathbf{W}$ by factor $1 / r_{i}^{\prime}$ to obtain $T_{1 / r_{i}^{\prime}}(\mathbf{W})$.

13. Calculate the NCC matrix (6) with $\mathbf{X}=\hat{\mathbf{K}}$ and $\mathbf{Y}=T_{1 / r_{i}^{\prime}}(\mathbf{Z}) T_{1 / r_{i}^{\prime}}(\mathbf{W})$.

14. Obtain $\operatorname{PCE}\left(r_{i}^{\prime}\right)$ according to (8).

15. If $\operatorname{PCE}\left(r_{i}^{\prime}\right)>\mathrm{PCE}_{\text {peak }}$, then $\mathrm{PCE}_{\text {peak }}=\operatorname{PCE}\left(r_{i}^{\prime}\right) ; r_{\text {peak }}=r_{i}^{\prime}$; end

end $\quad$ pphase 2 \}

16. If $\quad \mathrm{PCE}_{\text {peak }}>\tau$,

then begin

Declare the image source being identified.

Locate the maximum ( $\left.=\mathrm{PCE}_{\text {peak }}\right)$ in NCC to determine the cropping parameters $\left(u_{l}, u_{t}\right)=\mathbf{s}_{\text {peak }}$.

end

Output $\mathbf{s}_{\text {peak }}, r_{\text {peak }}$.

else Declare the image source unknown.

end

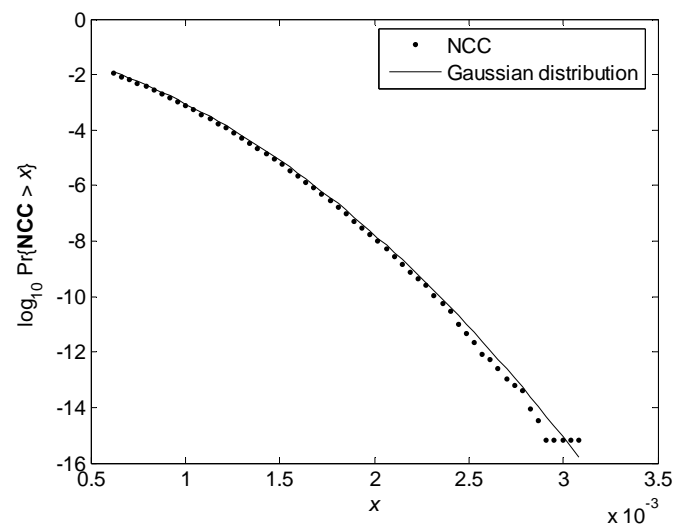

Fig. 2. Log-tail plot for the right tail of the sample distribution of NCC $\left[s ; r_{i}\right]$ for an unmatched case.

\subsection{Error analysis}

We make an assumption that, under hypothesis $\mathrm{H}_{0}$ for a fixed scaling ratio $r_{i}$, the values of the 2D normalized crosscorrelation $\mathbf{N C C}\left[\mathbf{s} ; r_{i}\right]$ as a function of $\mathbf{s}$ are i.i.d. samples of a Gaussian random variable $\zeta^{(i)} \sim N\left(0, \sigma_{i}^{2}\right)$. This is a plausible assumption due to the Central Limit Theorem and the fact that the two-dimensional signal $\mathbf{K}$ is wellmodeled as realizations of white Gaussian noise. Since the model fit at the tails is important for proper error analysis, 
we experimentally verified this assumption by providing in Fig. 2 the log-tail plot. It shows the probability $\log _{10} \operatorname{Pr}\{$ NCC $>x\}$ as a function of $x$ (continuous line) and the same probability calculated from the sample distribution of cross-correlations obtained experimentally for an incorrect scaling ratio for one selected test image. We can see that the right tail is consistent with the Gaussian assumption.

Estimating the variance $\sigma_{i}^{2}$ of the Gaussian model using the sample variance $\hat{\sigma}_{i}^{2}$ of $\mathbf{N C C}\left[\mathbf{s} ; r_{i}\right]$ over $\mathbf{s}$ after excluding a small central region $\mathcal{N}$ surrounding the peak

$$
\hat{\sigma}_{i}^{2}=\frac{1}{m n-|\mathcal{N}|} \sum_{\mathbf{s}, \mathbf{s} \in \mathcal{N}} \mathbf{N C C}\left[\mathbf{s} ; r_{i}\right]^{2},
$$

we now calculate the probability $p_{i}$ that $\zeta^{(i)}$ would attain the peak value $\mathbf{N C C}\left[\mathbf{s}_{\text {peak }} ; r_{\text {peak }}\right]$ or larger by chance. Using (8) and (10),

$$
p_{i}=\int_{\mathrm{NCC}\left[s_{\text {peak }} ; r_{\text {peak }}\right]}^{\infty} \frac{1}{\sqrt{2 \pi} \hat{\sigma}_{i}} e^{-\frac{x^{2}}{2 \hat{\sigma}_{i}^{2}}} d x=\int_{\hat{\sigma}_{\text {peak }} \sqrt{\mathrm{PCE}_{\text {peak }}}}^{\infty} \frac{1}{\sqrt{2 \pi} \hat{\sigma}_{i}} e^{-\frac{x^{2}}{2 \hat{\sigma}_{i}^{2}}} d x=Q\left(\frac{\hat{\sigma}_{\text {peak }}}{\hat{\sigma}_{i}} \sqrt{\mathrm{PCE}_{\text {peak }}}\right),
$$

where $Q(x)=1-\Phi(x)$ with $\Phi(x)$ denoting the cumulative distribution function of a normal variable $N(0,1)$ and $\mathrm{PCE}_{\text {peak }}=\operatorname{PCE}\left(r_{\text {peak }}\right)$. As explained in Section 2.1, during the search for the cropping vector $\mathbf{s}$, we only need to search in the range (7), which means that we are taking maximum over $k_{i}=\left(m-M / r_{i}+1\right) \times\left(n-N / r_{i}+1\right)$ samples of $\zeta^{(i)}$. Thus, the probability that the maximum value of $\xi^{(i)}$ would not exceed $\mathbf{N C C}\left[\mathbf{s}_{\text {peak }} ; r_{\text {peak }}\right]$ is $\left(1-p_{i}\right)^{k_{i}}$. After $R$ steps in the first phase of the search ${ }^{\ddagger}$ and $R^{\prime}$ steps in the second phase, the probability of false alarm, $P_{\mathrm{FA}}$, (deciding $\mathrm{H}_{1}$ while $\mathrm{H}_{0}$ is true) is

$$
P_{\mathrm{FA}}=1-\prod_{i=1}^{R+R^{\prime}}\left(1-p_{i}\right)^{k_{i}}
$$

Since in our algorithm, we stop the search after the PCE reaches a certain threshold, we have $r_{i} \leq r_{\text {peak }}$. Because $\hat{\sigma}_{i}^{2}$ is non-decreasing in $i, \hat{\sigma}_{\text {peak }} / \hat{\sigma}_{i} \geq 1$, and because $Q(x)$ is decreasing, we have $p_{i} \leq Q\left(\sqrt{\mathrm{PCE}_{\text {peak }}}\right)=p$. Thus, because $k_{i} \leq$ $m n$, from (12) we obtain an upper bound on $P_{\mathrm{FA}}$

$$
P_{\mathrm{FA}} \leq 1-(1-p)^{k_{\max }},
$$

where $k_{\max }=\sum_{i=0}^{R-1} k_{i}+\sum_{i=1}^{R^{\prime}} k_{i}^{\prime}$ is the maximal number of values of the parameters $r$ and $\mathbf{s}$ over which the maximum of (6) could be taken, $k_{i}^{\prime}=\left(m-M / r_{i}^{\prime}+1\right) \times\left(n-N / r_{i}^{\prime}+1\right)$.

The threshold for PCE, $\tau=\tau\left(P_{\mathrm{FA}}, M, N, m, n\right)$, depends on the probability of false alarm $P_{\mathrm{FA}}$ and the size of the search space for the parameters, which is determined by the dimensions of PRNU and the dimensions of the image under investigation. For example, for our tested Canon G2 camera with $m=1704$ and $n=2272$, image size $M=833$, $N=1070, P_{\mathrm{FA}} \leq 10^{-5}, R=201$ according to Step 2 and $R^{\prime}=12$ for Phase 2 of the search, $k_{\max }=88,836,025$ and the threshold for PCE is $\tau=53.772$.

\section{EXPERIMENTAL RESULTS}

Three types of experiments are presented in this section. The scaling only and cropping only experiments performed on 5 different test images along with (and without) JPEG compression are in Section 3.1. Section 3.2 contains random cropping and random scaling tests with JPEG compression on a single image. This test follows the most likely "real life" scenario and reveals how each processing step affects camera identification. In Section 3.3, we discuss a special case of cropping and scaling which occurs when digital zoom is engaged in the camera.

${ }^{\ddagger}$ Recall that the parameter $R$ is the number of samples used in Phase 1 of the search for the scaling parameter. 


\subsection{Scaling only and cropping only}

Fig. 3 shows five test images from Canon G2 with a 4 Mp CCD sensor. These images cover a wide range of difficulties from the point of view of camera identification with the first one being the easiest because it contains large flat and bright areas and the last one the most difficult due to its rich texture. ${ }^{17}$ The camera fingerprint $\mathbf{K}$ was estimated from 30 blue sky images in the TIFF format. It was also preprocessed using the column and row zeromeaning ${ }^{17}$ to remove any residual patterns not unique to the sensor. This step is important because periodicities in demosaicking errors would cause unwanted interference at certain translations and scaling factors, consequently decreasing the PCE and increasing the false alarm rate. We found that this effect can be quite substantial.

We performed several different tests to first gain insight into how robust the camera ID algorithm is. In the Scaling Only Test, we only subjected the test images to scaling with progressively smaller scaling parameter $r$ and displayed the results in Table 1 showing the PCE( $r$ ) for $0.3 \leq r \leq 0.9$, with no lossy compression and with JPEG compression with quality factors $90 \%, 75 \%$, and $60 \%$. The downsampling method was bicubic resampling. The upsampling used in our search algorithm was the nearest neighbor algorithm. Here, we intentionally used a different resampling algorithm because in reality we will not know the resampling algorithm and we want the tests to reflect real life conditions.

In the Cropping Only Test, all images were only subjected to cropping with an increasing amount of the cropped out region. The cropped part was always the lower-right corner of the images. We note that while scaling by the ratio $r$ means that the image dimensions were scaled by the factor $r$, cropping by a factor $r$ means that the size of the cropped image is $r$ times the original dimension. In particular, scaling and cropping by the same factor produces images with the same number of pixels, $r^{2} \times m n$.

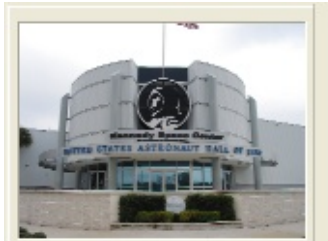

(1)

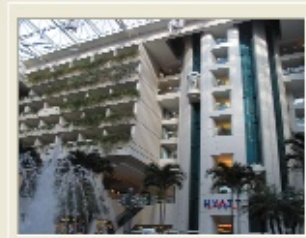

(2)

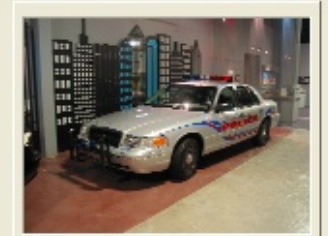

(3)

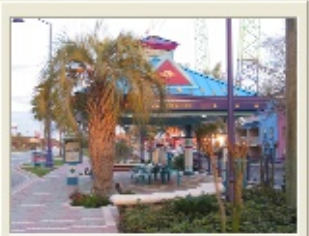

(4)

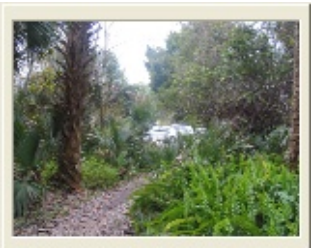

(5)

Fig. 3. Five test images from a 4 MP Canon G2 camera ordered by the richness of their texture (their difficulty to be identified).

The image identification in the Scaling Only Test (left half of Table 1) was successful for all 5 images and JPEG compression factors when the scaling factor was not smaller than 0.5. It started failing when the scaling ratio was 0.4 or lower and the JPEG quality was $75 \%$ or lower. Image \#5 was correctly identified at ratios 0.5 and above although its content is difficult to suppress for the denoising filter. ${ }^{17}$ The largest PCE that did not determine the correct parameters [ $\left.\mathbf{s}_{\text {peak }} ; r_{\text {peak }}\right]$ was 38.502 (image \#1). On the other hand, the lowest PCE for which the parameters were correctly determined was 35.463 (also for image \#1). We encountered some cases when the maximum value of NCC did occur for the correct cropping and scaling parameters but the identification algorithm failed because the PCE was below the threshold set to achieve $P_{\mathrm{FA}} \leq 10^{-5}$.

Image cropping has a much smaller effect on image identification (the Cropping Only Test part of Table 1). We correctly determined the exact position of the cropped image within the (unknown) original in all tested cases. The PCE was consistently above 130 even when the images were cropped to the small size $0.3 m \times 0.3 n$ and compressed with JPEG quality factor 60 .

\subsection{Random cropping and random scaling simultaneously}

This series of tests focused on the performance of the search method on image \#2. The image underwent 50 simultaneous random cropping and scaling with both scaling and cropping ratios between 0.5 and 1 followed by JPEG compression with the same quality factors as in the previous tests. We sorted the maximum PCE values found in each search by the scaling ratio (since it has by far the biggest influence on the algorithm performance) and plotted the PCE in Fig. 4. The threshold $\tau=56.315$ displayed in the figure corresponds to the worst scenario (largest search space) of 0.5 scaling and 0.5 cropping for false alarm rate below $10^{-5}$. In the test, we did not observe any missed detection for the JPEG quality factor 90, 1 missed detection for JPEG quality factor 75 and scaling ratio close to 0.5 , and 5 missed 
detections for JPEG quality factor 60 when the scaling ratios were below 0.555 . Even though these numbers will vary significantly with the image content, they give us some insight into the robustness of the proposed method.

The last test was a large scale test intended to evaluate the real-life performance of the proposed methodology. The database of 720 images contained snapshots spanning the period of four years. All images were taken at the full CCD resolution and with a high JPEG quality setting. Each image was first subjected to a randomly-generated cropping up to $50 \%$ in each dimension. The cropping position was also chosen randomly with uniform distribution within the image. The cropped part was further resampled by a scaling ratio $r \in[0.5,1]$. Finally, the image was compressed with 85\% quality JPEG. The false alarm was set again to $10^{-5}$. Running our algorithm with $r_{\min }=0.5$ on all images processed this way, we encountered 2 missed detections (Fig. 5a), which occurred for more difficult (textured) images. In all successful detections, the cropping and scaling parameters were detected with accuracy better than 2 pixels in either dimension.

To complement this test, 915 images from more than 100 different cameras were downloaded from the Internet in native resolution, cropped to the $4 \mathrm{Mp}$ size of Canon G2 and subjected to the same random cropping, scaling, and JPEG compression as the 720 images before. No single false detection was encountered. All maximum values of PCE were below the threshold with the overall maximum at 42.5 .

Table 1. PCE in the Scaling Only Test followed by JPEG compression. The PCE is in italic when the scaling ratio was not determined correctly. Values in parentheses are below the detection threshold $\tau$ (see the leftmost column) for $P_{\mathrm{FA}}<10^{-5}$.

\begin{tabular}{|c|c|c|c|c|c|c|c|c|c|c|c|}
\hline & & \multicolumn{5}{|c|}{ Scaling Only Test } & \multicolumn{5}{|c|}{ Cropping Only Test } \\
\hline & & Im. \#1 & Im. \#2 & Im. \#3 & Im. \#4 & Im. \#5 & Im. \#1 & Im. \#2 & Im. \#3 & Im. \#4 & Im. \#5 \\
\hline & TIFF & 68003 & 32325 & 28570 & 20092 & 2964 & 14624 & 9157 & 7789 & 8961 & 3106 \\
\hline$r=0.9$ & Q 90 & 28951 & 12834 & 11347 & 6971 & 1515 & 20905 & 11475 & 8223 & 7348 & 2637 \\
\hline \multirow[t]{2}{*}{$\tau=45.1$} & Q 75 & 6131 & 3343 & 3436 & 2225 & 793 & 7192 & 4509 & 4668 & 3193 & 1480 \\
\hline & Q 60 & 1778 & 1245 & 1291 & 1037 & 461 & 2751 & 2029 & 2227 & 1706 & 919 \\
\hline \multirow{4}{*}{$\begin{aligned} r & =0.8 \\
\tau & =48.3\end{aligned}$} & TIFF & 72971 & 35086 & 30128 & 17494 & 2260 & 9282 & 8148 & 7764 & 8043 & 3058 \\
\hline & Q 90 & 19041 & 8045 & 7080 & 4171 & 955 & 15287 & 8988 & 8251 & 6060 & 2329 \\
\hline & Q 75 & 2980 & 1610 & 1736 & 1115 & 417 & 5698 & 2979 & 3977 & 2550 & 1226 \\
\hline & Q 60 & 763 & 645 & 614 & 348 & 222 & 2216 & 1224 & 1916 & 1337 & 754 \\
\hline \multirow{4}{*}{$\begin{array}{l}r=0.7 \\
r=\mathrm{P} 50.4\end{array}$} & TIFF & 41835 & 20058 & 17549 & 9674 & 910 & 6496 & 7986 & 7384 & 6030 & 2508 \\
\hline & Q 90 & 8255 & 3642 & 3731 & 1938 & 410 & 11434 & 7887 & 9210 & 4533 & 2037 \\
\hline & Q 75 & 1316 & 724 & 979 & 507 & 170 & 4565 & 2593 & 3807 & 2142 & 995 \\
\hline & Q 60 & 328 & 318 & 406 & 203 & 124 & 1774 & 1155 & 1860 & 1139 & 622 \\
\hline \multirow{4}{*}{$\begin{array}{l}r=0.6 \\
\tau=52.1\end{array}$} & TIFF & 42192 & 18991 & 16902 & 8986 & 1001 & 4896 & 8249 & 6550 & 4353 & 1971 \\
\hline & Q 90 & 4619 & 2060 & 2195 & 1311 & 422 & 7679 & 6328 & 8574 & 2918 & 1445 \\
\hline & Q 75 & 625 & 399 & 476 & 214 & 117 & 2883 & 1951 & 3443 & 1255 & 682 \\
\hline & Q 60 & 190 & 166 & 196 & 85 & 53 & 1166 & 709 & 1568 & 669 & 459 \\
\hline \multirow{4}{*}{$\begin{array}{l}r=0.5 \\
\tau=53.5\end{array}$} & TIFF & 24767 & 10719 & 9442 & 4781 & 487 & 4339 & 6592 & 5216 & 3006 & 1599 \\
\hline & Q 90 & 1721 & 997 & 927 & 533 & 196 & 6314 & 3989 & 6996 & 2206 & 1265 \\
\hline & Q 75 & 211 & 168 & 221 & 134 & 111 & 2622 & 1089 & 2799 & 1003 & 695 \\
\hline & Q 60 & 144 & 56 & 70 & (50) & 58 & 1216 & 398 & 1322 & 574 & 464 \\
\hline \multirow{4}{*}{$\begin{array}{l}r=0.4 \\
\tau=54.8\end{array}$} & TIFF & 8083 & 3193 & 2897 & 1310 & 123 & 4008 & 4469 & 4163 & 1881 & 1086 \\
\hline & Q 90 & 457 & 227 & 280 & 151 & (52) & 3730 & 2274 & 5178 & 1230 & 846 \\
\hline & Q 75 & 72 & (52) & 72 & (53) & 31 & 1407 & 723 & 2113 & 570 & 453 \\
\hline & Q 60 & (35) & (43) & (43) & 26 & 34 & 599 & 255 & 1006 & 311 & 324 \\
\hline \multirow{4}{*}{$\begin{array}{l}r=0.3 \\
\tau=55.9\end{array}$} & TIFF & 777 & 352 & 477 & 124 & 27 & 3518 & 1585 & 3030 & 916 & 837 \\
\hline & Q 90 & (46) & (41) & 60 & 38 & 30 & 2577 & 742 & 3414 & 657 & 609 \\
\hline & Q 75 & 39 & 32 & 35 & 31 & 35 & 969 & 269 & 1378 & 300 & 259 \\
\hline & Q 60 & 35 & 33 & 35 & 32 & 35 & 461 & 139 & 721 & 164 & 177 \\
\hline
\end{tabular}




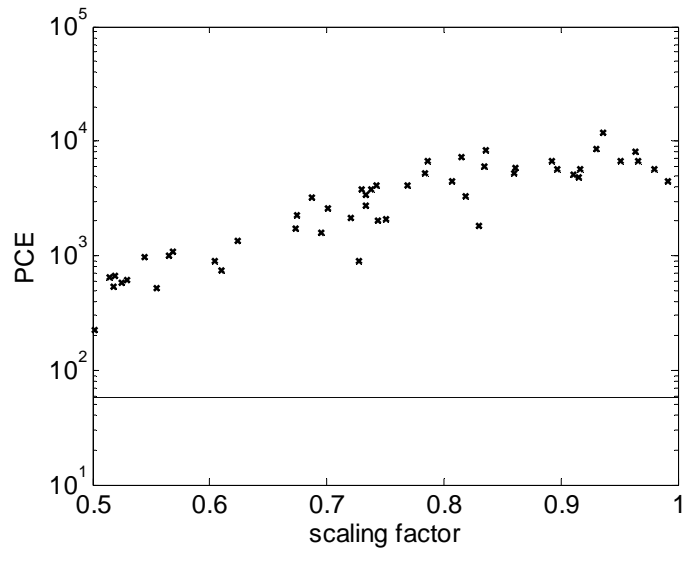

(a) JPEG quality factor 90

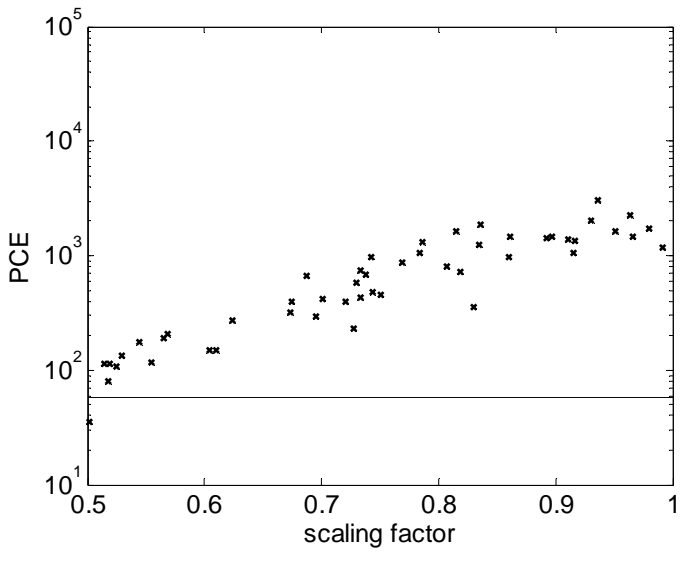

(b) JPEG quality factor 75

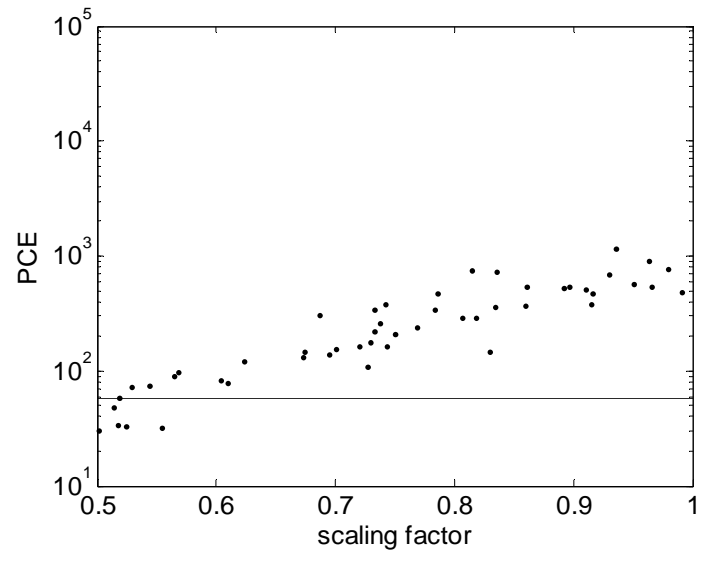

(c) JPEG quality factor 60

Fig. 4. PCE for image \#2 after a series of random scaling and cropping followed by $90 \%$ quality JPEG compression.

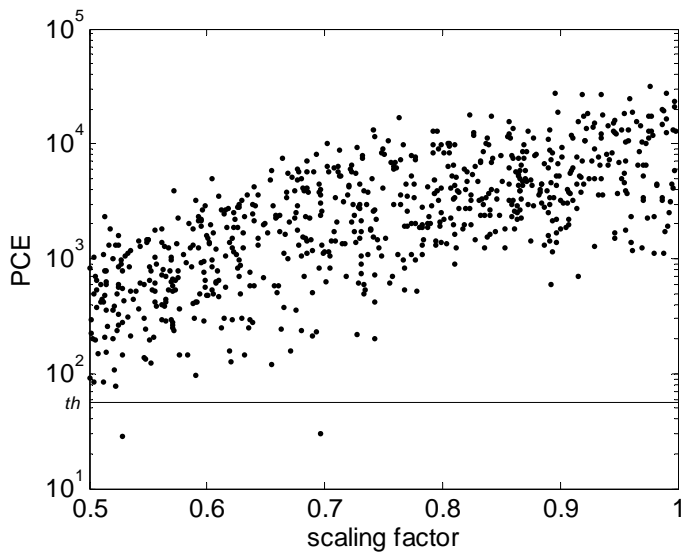

(a) PCE as a function of the scaling ratio for 720 images matching the camera.

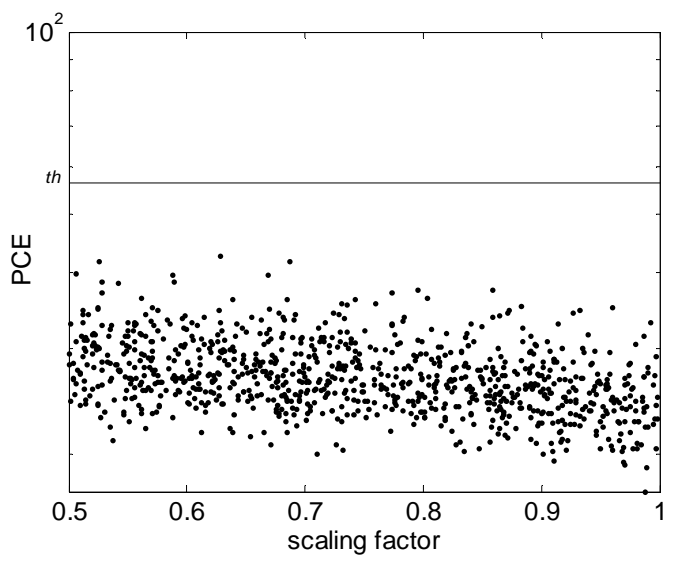

(b) PCE for 915 images not matching the camera.

Fig. 5. "Real-life large scale test” for JPEG images subjected to random cropping and scaling and saved as 85\% quality JPEG. The lines mark the threshold for $P_{\mathrm{FA}} \leq 10^{-5}$ ( $\tau=56.315$ ). (a) 720 images from the camera, (b) 915 images from other cameras. 


\subsection{Digital zoom}

While optical zoom does not desynchronize PRNU with the image noise residual (it is equivalent to a change of scene), when a camera engages digital zoom, it introduces the following geometrical transformation: the middle part of the image is cropped and up-sampled to the resolution determined by the camera settings. This is a special case of our cropping and scaling scenario. Since the cropping may be a few pixels off the center, we need to search for the scaling factor $r$ as well as the shift vector $\mathbf{s}$. The maximum digital zoom determines the upper bound on our search for the scaling factor. For simplicity, we apply the same search for cropping as before although we could restrict ourselves to a smaller search range around the image center.

Some cameras allow almost continuous digital zoom (e.g., Fuji E550) while other offer only several fixed values. This is the case of Canon S2 that we tested. The camera display indicates zoom values " $24 \times$ ”, “30×”, "37×”, and " $48 \times$ ”, which correspond to digital zoom scaling ratios $1 / 2,1 / 2.5,1 / 3.0833$, and $1 / 4$, considering the $12 \times$ camera optical zoom. Our test revealed exact scaling ratios $1 / 2.025,1 / 2.5313,1 / 3.1154$, and $1 / 4$, corresponding to cropped sizes $1280 \times 960,1024 \times 768,832 \times 624$, and $648 \times 486$, respectively. Thus, in general for camera identification, we may wish to check these digital zoom scaling values first before proceeding with the rest of the search if no match is found. We note that none of the camera manuals for the two tested cameras (Fuji and Canon) contained any information about the digital zoom. We only found out the details about their digital zooms using the PRNU! Thus, this is an interesting example of using the PRNU as a template to recover processing history or reverse-engineer in-camera processing.

Table 2 shows the maximal PCE on 10 images taken with Canon S2 and Fuji E550, some of which were taken with digital zoom. Both cameras were identified with very high confidence in all 10 cases. Images from Fuji camera yielded smaller maximum PCEs, which suggests that (if the image content is dark or heavily textured) the identification of Fuji E550 camera could be more difficult than Canon S2. The detected cropped dimensions (see Table 2) are either precise or off only by a few pixels. This camera apparently has a much finer increment when adjusting the digital zoom than Canon S2. Since the Fuji E550 user is not informed about the fact that the digital zoom has been engaged, it may be quite tedious to find all possible scaling values in this case. The largest digital zoom the camera offers for full resolution output size is 1.4. Fig. 6 shows images with detected cropped frame for the last two Fuji camera images of the same scene.

The fact that we are able to obtain previous dimensions of the up-sampled images is an example of "reverse engineering" for revealing image processing history. Such information is potentially useful in forensics sciences even if the source camera is positively known beforehand.

Table 2. Detection of scaling and cropping for digitally zoomed images.

\begin{tabular}{|c|c|c|c|r|r|c|}
\hline & \multicolumn{3}{|c|}{ Canon S2 } & \multicolumn{3}{c|}{ Fuji E550 } \\
\hline $\begin{array}{c}\text { Image } \\
\#\end{array}$ & $\begin{array}{c}\text { scaling } \\
\text { detected }\end{array}$ & $\begin{array}{c}\text { max } \\
\text { PCE }\end{array}$ & $\begin{array}{c}\text { cropped } \\
\text { dim }\end{array}$ & $\begin{array}{c}\text { scaling } \\
\text { detected }\end{array}$ & $\begin{array}{c}\text { max } \\
\text { PCE }\end{array}$ & $\begin{array}{c}\text { cropped } \\
\text { dim }\end{array}$ \\
\hline 1 & 3.1154 & 1351 & $832 \times 624$ & 1.1530 & 358 & $2470 \times 1853$ \\
2 & 2.5313 & 6020 & $1024 \times 768$ & 1.3434 & 238 & $2120 \times 1590$ \\
3 & 2.0250 & 2792 & $1280 \times 960$ & 1.3940 & 102 & $2043 \times 1532$ \\
4 & 2.0203 & 9250 & $1283 \times 962$ & 1.1837 & 310 & $2406 \times 1805$ \\
5 & 2.5313 & 5929 & $1024 \times 768$ & 1.0234 & 576 & $2783 \times 2087$ \\
6 & 3.1154 & 3509 & $832 \times 624$ & 1.0007 & 328 & $2846 \times 2135$ \\
7 & 4.0062 & 2450 & $647 \times 485$ & 1.0000 & 314 & $2848 \times 2136$ \\
8 & 4.0062 & 1265 & $647 \times 485$ & 1.0845 & 1022 & $2626 \times 1970$ \\
9 & 4.0062 & 1620 & $649 \times 487$ & 1.1530 & 976 & $2470 \times 1853$ \\
10 & 4.0062 & 1612 & $647 \times 485$ & 1.3428 & 378 & $2121 \times 1591$ \\
\hline
\end{tabular}



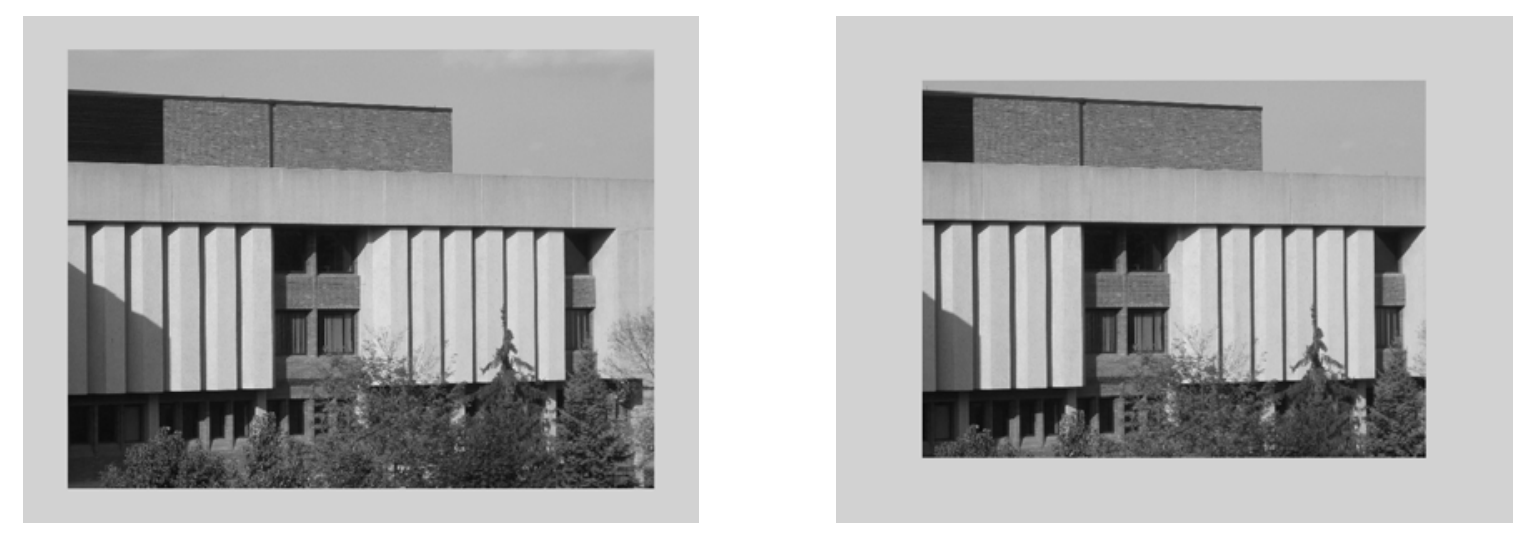

Fig. 6. Cropping detected for Fuji E550 images \#9 and \#10.

\section{CONCLUSIONS}

In this paper, we present an extension of our previous work on camera identification from images using sensor pattern noise. The proposed method is capable of identifying the camera after the image under investigation was simultaneously cropped and scaled or when the image was taken with a digital zoom. The camera ID is formulated as a two-channel detection problem. The detector is obtained using the generalized likelihood ratio test and has a form of a cross-correlation maximized over the parameters of the geometrical transform. In fact, we recommend using crosscorrelation and the PCE instead of the correlation as the detection statistics even for images that did not undergo any geometrical processing because the cross-correlation surface enables more accurate error rate estimation. We report the results for a wide range of scaling and cropping parameters and JPEG compression. The results indicate that reliable camera ID is possible for images linearly scaled down by a factor of 0.5 or more or images with $90 \%$ or more cropped away. The reliability depends on the image content and subsequent JPEG compression. We also demonstrate that the sensor noise can be used as a template to reverse-engineer in-camera geometrical processing, such as processing due to digital zoom. We acknowledge that the proposed method can be computationally expensive, namely for large image dimensions. A hierarchical search that starts with a large step which is then decreased by $1 / 2$ in each round makes the search process about 4 times faster, however only if the match is positive.

\section{ACKNOWLEGEMENT}

The work on this paper was supported by the AFOSR grant number FA9550-06-1-0046. The U.S. Government is authorized to reproduce and distribute reprints for Governmental purposes notwithstanding any copyright notation thereon. The views and conclusions contained herein are those of the authors and should not be interpreted as necessarily representing the official policies, either expressed or implied, of Air Force Research Laboratory, or the U.S. Government.

\section{REFERENCES}

1. S. Bayram, H.T. Sencar, and N. Memon: "Source Camera Identification Based on CFA Interpolation." Proc. ICIP'05, Genoa, Italy, September 2005.

2. A. Swaminathan, M. Wu, and K.J.R. Liu: "Non-intrusive Forensic Analysis of Visual Sensors Using Output Images.” Proc. IEEE Int. Conf. on Acoustics, Speech, and Signal Processing (ICASSP'06), May 2006.

3. A. Swaminathan, M. Wu, and K.J.R. Liu: "Image Authentication via Intrinsic Fingerprints." Proc. SPIE, Electronic Imaging, Security, Steganography, and Watermarking of Multimedia Contents IX, vol. 6505, January 29-February 1, San Jose, CA, pp. 1J-1K, 2007.

4. M. Kharrazi, H.T. Sencar, and N. Memon: "Blind Source Camera Identification,” Proc. ICIP'04, Singapore, October 24-27, 2004. 
5. K. Kurosawa, K. Kuroki, and N. Saitoh: "CCD Fingerprint Method - Identification of a Video Camera from Videotaped Images.” Proc. ICIP’99, Kobe, Japan, pp. 537-540, October 1999.

6. Z. Geradts, J. Bijhold, M. Kieft, K. Kurosawa, K. Kuroki, and N. Saitoh: "Methods for Identification of Images Acquired with Digital Cameras." Proc. of SPIE, Enabling Technologies for Law Enforcement and Security, vol. 4232, pp. 505-512, February 2001.

7. J. Lukáś, J. Fridrich, and M. Goljan: "Digital Camera Identification from Sensor Pattern Noise." IEEE Transactions on Information Security and Forensics, vol. 1(2), pp. 205-214, June 2006.

8. H. Gou, A. Swaminathan, and M. Wu: "Robust Scanner Identification Based on Noise Features." Proc. SPIE, Electronic Imaging, Security, Steganography, and Watermarking of Multimedia Contents IX, vol. 6505, January 29-February 1, San Jose, CA, pp. 0S-0T, 2007.

9. N. Khanna, A.K. Mikkilineni, G.T.C Chiu, J.P. Allebach, and E.J. Delp III: "Forensic Classification of Imaging Sensor Types." Proc. SPIE, Electronic Imaging, Security, Steganography, and Watermarking of Multimedia Contents IX, vol. 6505, January 29-February 1, San Jose, CA, pp. 0U-0V, 2007.

10. B. Sankur, O. Celiktutan, and I. Avcibas: "Blind Identification of Cell Phone Cameras." Proc. SPIE, Electronic Imaging, Security, Steganography, and Watermarking of Multimedia Contents IX, vol. 6505, January 29February 1, San Jose, CA, pp. 1H-1I, 2007.

11. T. Gloe, E. Franz, and A. Winkler: "Forensics for Flatbed Scanners." Proc. SPIE, Electronic Imaging, Security, Steganography, and Watermarking of Multimedia Contents IX, vol. 6505, January 29-February 1, San Jose, CA, pp. 1I-1J, 2007.

12. N. Khanna, A.K. Mikkilineni, G.T.C. Chiu, J.P. Allebach, and E.J. Delp III: "Scanner Identification Using Sensor Pattern Noise." Proc. SPIE, Electronic Imaging, Security, Steganography, and Watermarking of Multimedia Contents IX, vol. 6505, January 29-February 1, San Jose, CA, pp. 1K-1L, 2007.

13. C.W. Honsinger and S.J. Daly: "Method for Detecting Rotation and Magnification in Images," United States Patent 5,835,639, Kodak, 1998.

14. N. Nikolaidis and I. Pitas: "Circularly Symmetric Watermark Embedding in 2-D DFT Domain,” In: Proc. IEEE Int. Conf. on Acoustics, Speech, and Signal Processing (ICASSP99), vol. 6, pp. 3469-3472, Phoenix, AZ, March 15-19, 1999.

15. J.J.K. Ó Ruanaidh and T. Pun: "Rotation, Scale and Translation Invariant Spread Spectrum Digital Image Watermarking,” Signal Processing, vol. 66(5), pp. 303-317, May 1998.

16. A.C. Popescu and H. Farid: "Exposing Digital Forgeries by Detecting Traces of Resampling." IEEE Transactions on Signal Processing, vol. 53(2), pp. 758-767, 2005.

17. J. Fridrich, M. Chen, M. Goljan, and J. Lukáš: "Digital Imaging Sensor Identification (Further Study),” Proc. SPIE, Electronic Imaging, Security, Steganography, and Watermarking of Multimedia Contents IX, vol. 6505, San Jose, CA, January 28-February 2, pp. 0P-0Q, 2007.

18. M.K. Mihcak, I. Kozintsev, and K. Ramchandran: "Spatially Adaptive Statistical Modeling of Wavelet Image Coefficients and its Application to Denoising," Proc. IEEE Int. Conf. Acoustics, Speech, and Signal Processing, Phoenix, Arizona, vol. 6, pp. 3253-3256, March 1999.

19. C.R. Holt: "Two-Channel Detectors for Arbitrary Linear Channel Distortion," IEEE Trans. on Acoustics, Speech, and Sig. Proc., vol. ASSP-35(3), pp. 267-273, March 1987.

20. B.V.K. Vijaya Kuma and L. Hassebrook: "Performance Measures for Correlation Filters,” Appl. Opt. 29, 29973006, 1990.

21. S.M. Kay, Fundamentals of Statistical Signal Processing, Volume II, Detection theory, Prentice Hall, 1998. 